\title{
Dual-Redox System of Metallo-Supramolecular Polymers for Visible-to-Near-IR Modulable Electrochromism and Durable Device Fabrication
}

Sanjoy Mondal, Dines Chandra Santra, Yoshikazu Ninomiya, Takefumi Yoshida and Masayoshi Higuchi*

Electronic Functional Macromolecules Group, National Institute for Materials Science (NIMS), 1-1 Namiki, Tsukuba 305-0044, Japan,

E-mail: HIGUCHI.Masayoshi@ @ims.go.jp 


\section{Experimental}

Instruments: The ${ }^{1} \mathrm{H}$ and ${ }^{13} \mathrm{C}$ spectra were recorded using JEOL AL 300/BZ (400 MHz) instrument. The molecular wight was measured using a MASS timsTOF spectrometer (Bruker, Version 2.2). The UV-vis study was performed using Shimadzu UV-visible spectrophotometer (UV-2550). All electrochemical measurements were performed using an ALS/CHI electrochemical workstation (CH Instruments, Inc.) integrated with an Ocean Optics modular to monitor the optical changes. Thermogravimetric analysis (TGA) was measured using TG/DTA 6200 instrument (Seiko) in a $\mathrm{N}_{2}$ environment with a $10^{\circ} \mathrm{C} / \mathrm{min}$ heating rate. SEC-viscometry-RALS (size exclusion chromatography-viscometry-right angle light scattering solvent) with polyethylene oxide-PEO-19K as standard was used for molecular weight determination of polymers. 


\section{The ${ }^{1} \mathrm{H}$ - and ${ }^{13} \mathrm{C}$-NMR spectra of $L_{\text {TPN }}$}

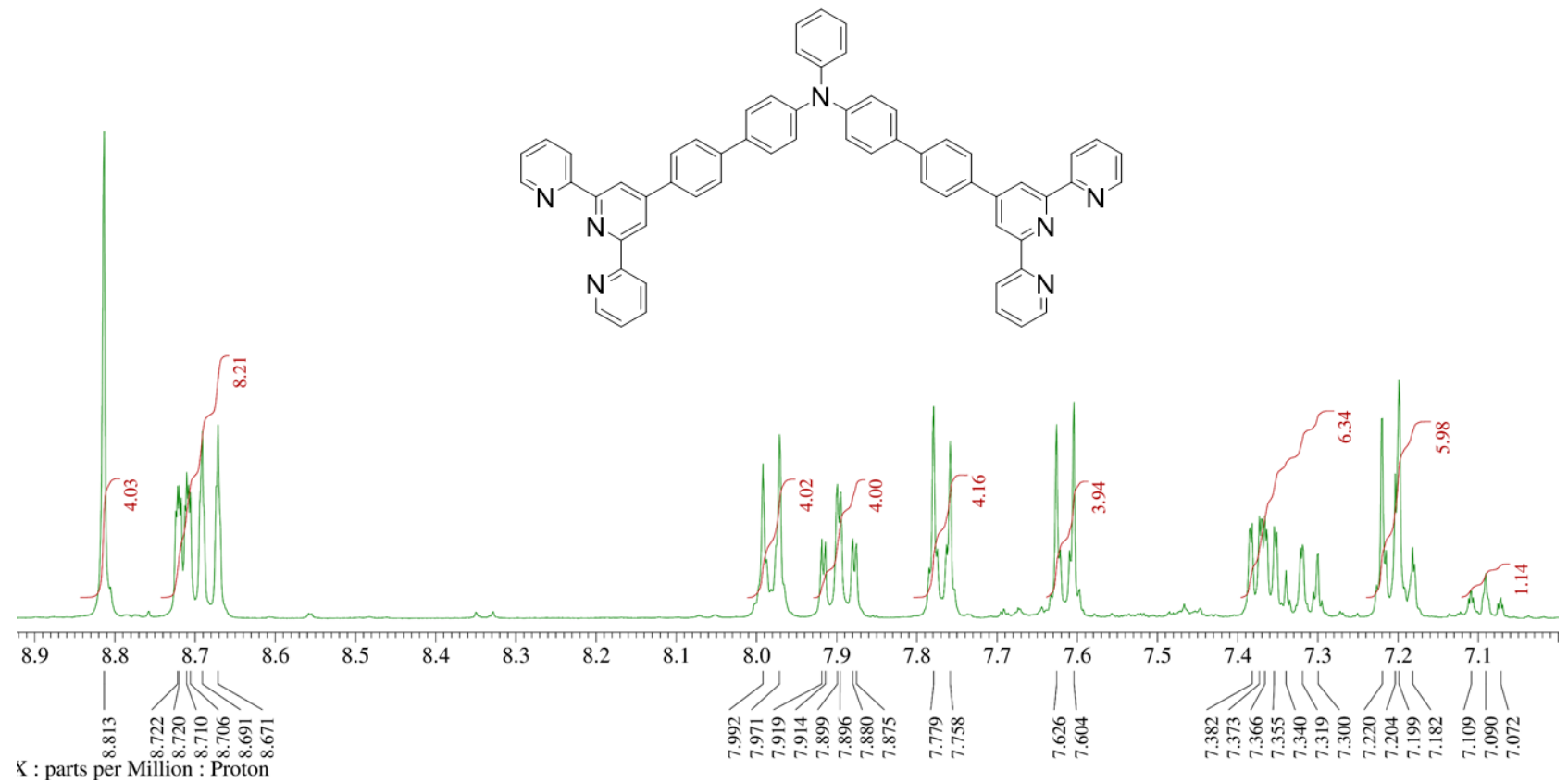

Figure S1. The ${ }^{1} \mathrm{H}-\mathrm{NMR}$ spectrum of $\mathbf{L}$ TPA $\left(400 \mathrm{MHz}, \mathrm{CD}_{2} \mathrm{Cl}_{2}\right)$.

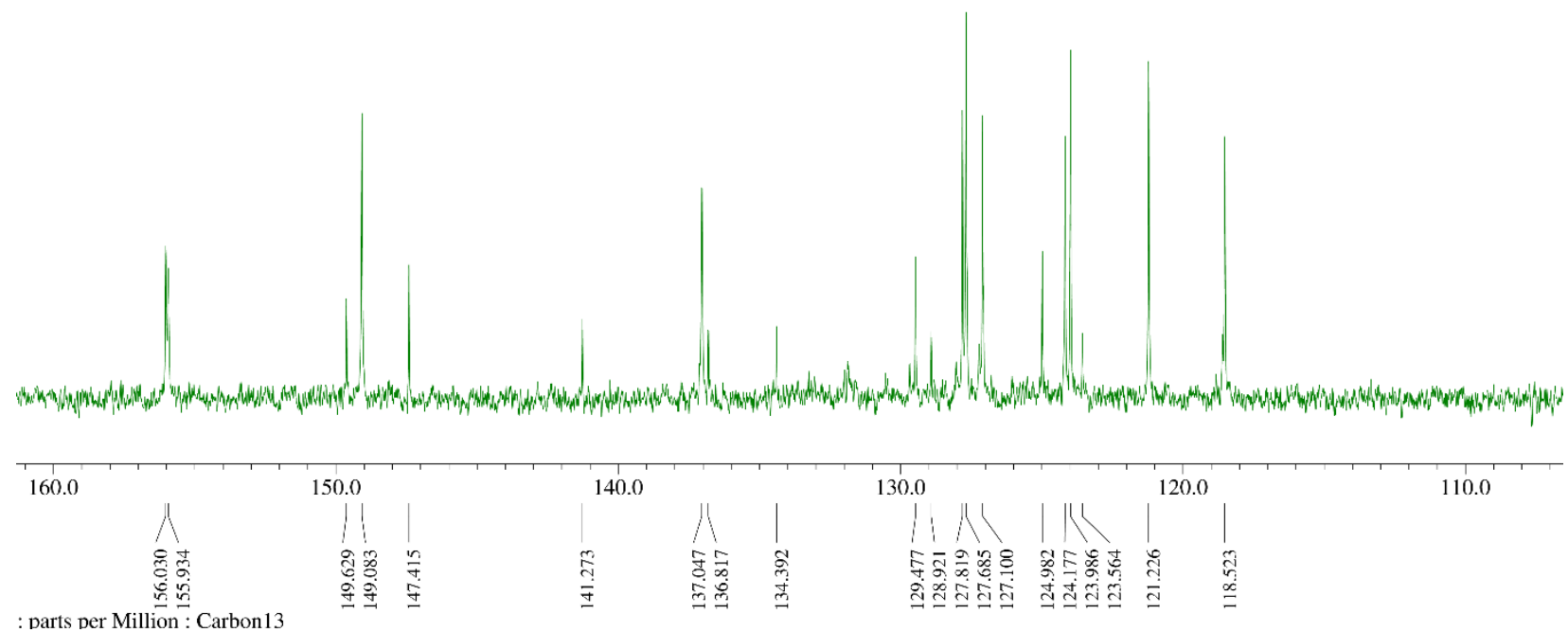

Figure S2. The ${ }^{13} \mathrm{C}-\mathrm{NMR}$ spectrum of $\mathbf{L T P A}\left(100 \mathrm{MHz}, \mathrm{CD}_{2} \mathrm{Cl}_{2}\right)$. 


\section{ESI-MS study of LTPN}

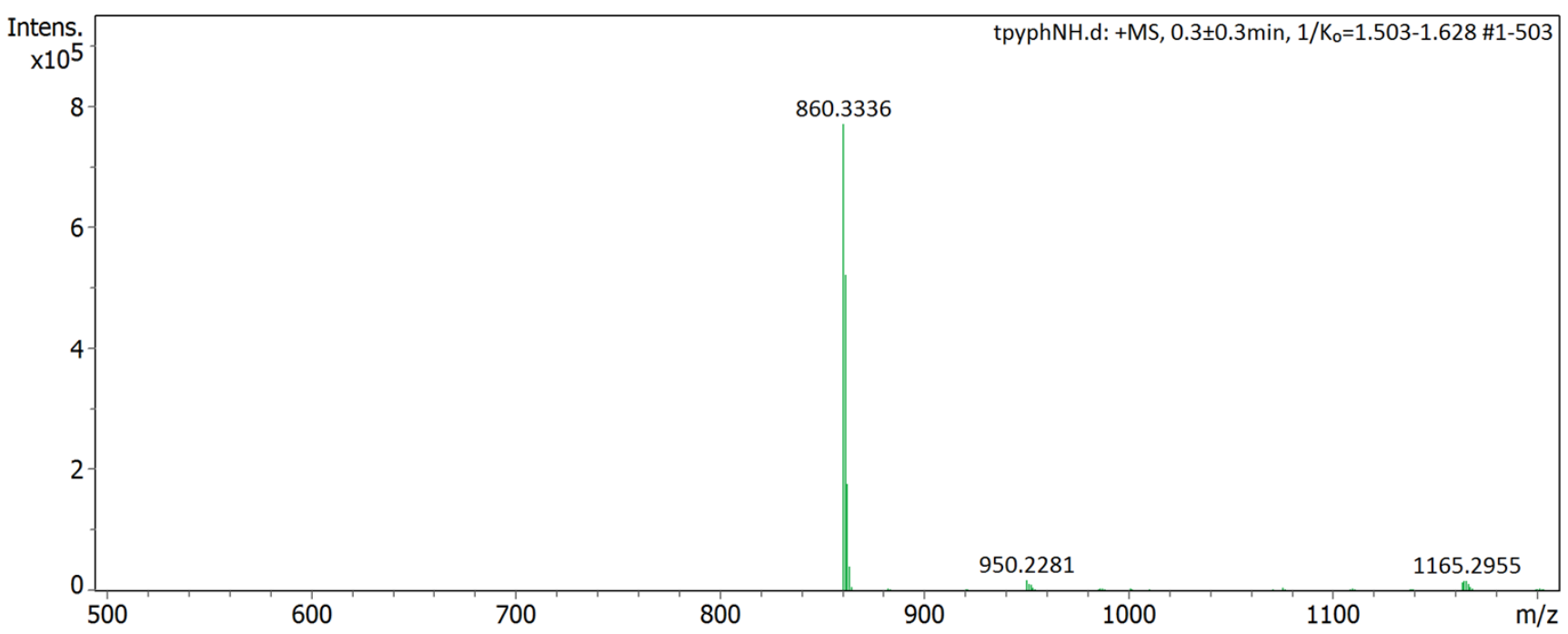

Figure S3. ESI-TOF-MS spectrum of LTPA.

\section{4. ${ }^{1} H-N M R$ spectra of LTPA and polymers}

Figure S4. The ${ }^{1} \mathrm{H}$ NMR spectra of ligand (LTPA), polyFe-N and polyRu-N.
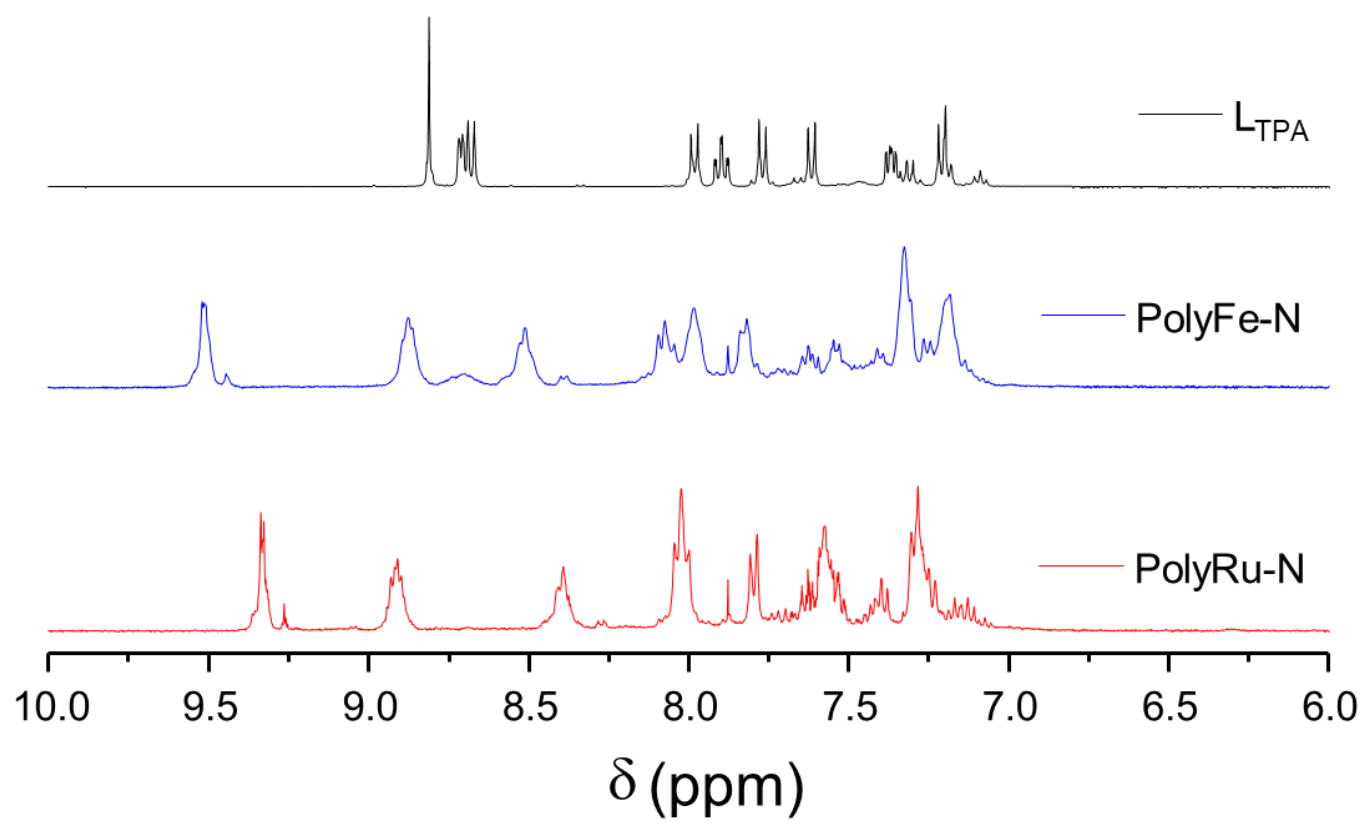


\section{XPS study}

(a)

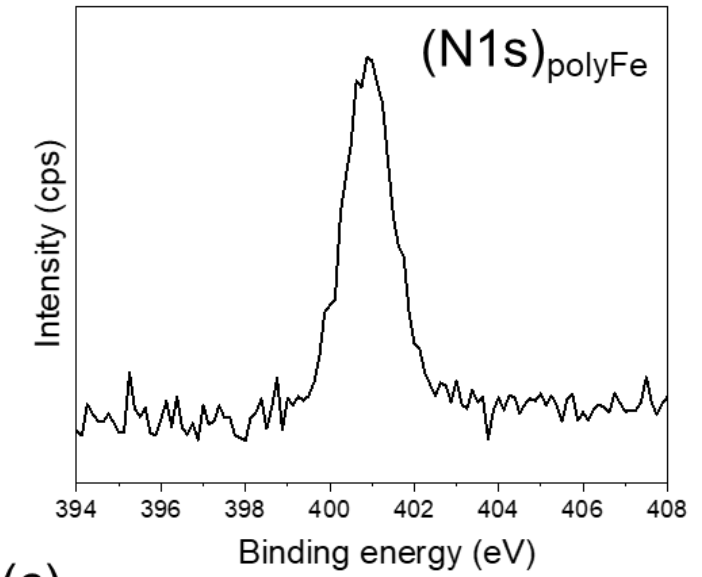

(c)

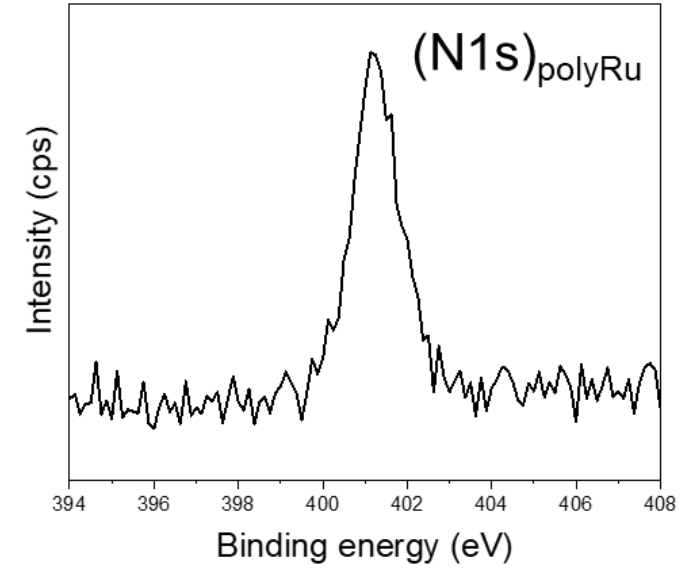

(b)

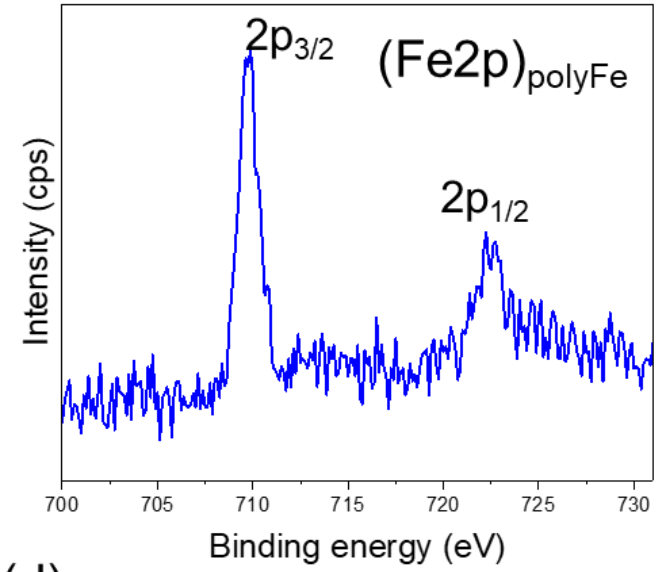

(d)

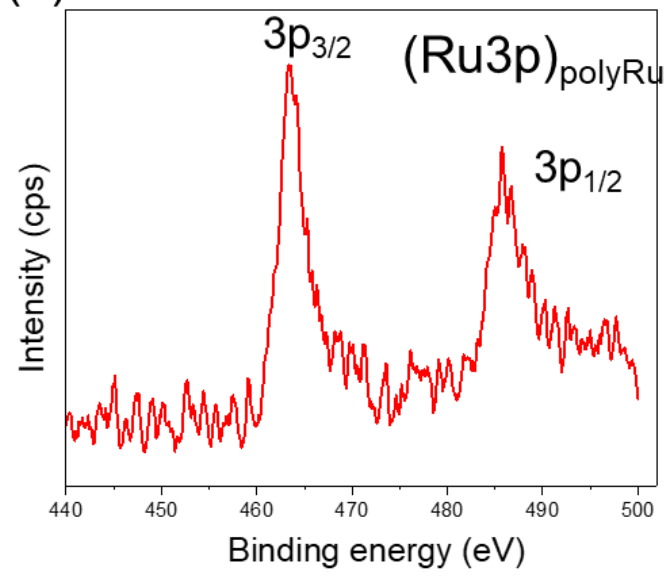

Figure S5. In the XPS study, the high-resolution scan for (a) N1s and (b) Fe2p for polyFe-N; (c) N1s and (d) Ru3p for polyRu-N. 


\section{AFM height profile diagram of polymer film}

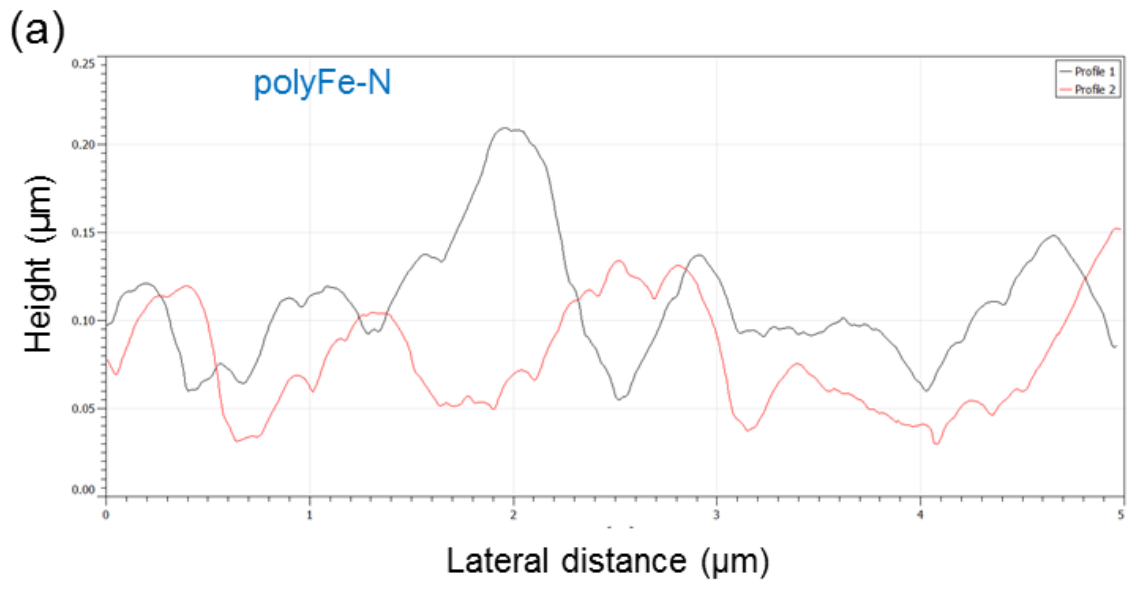

(b)

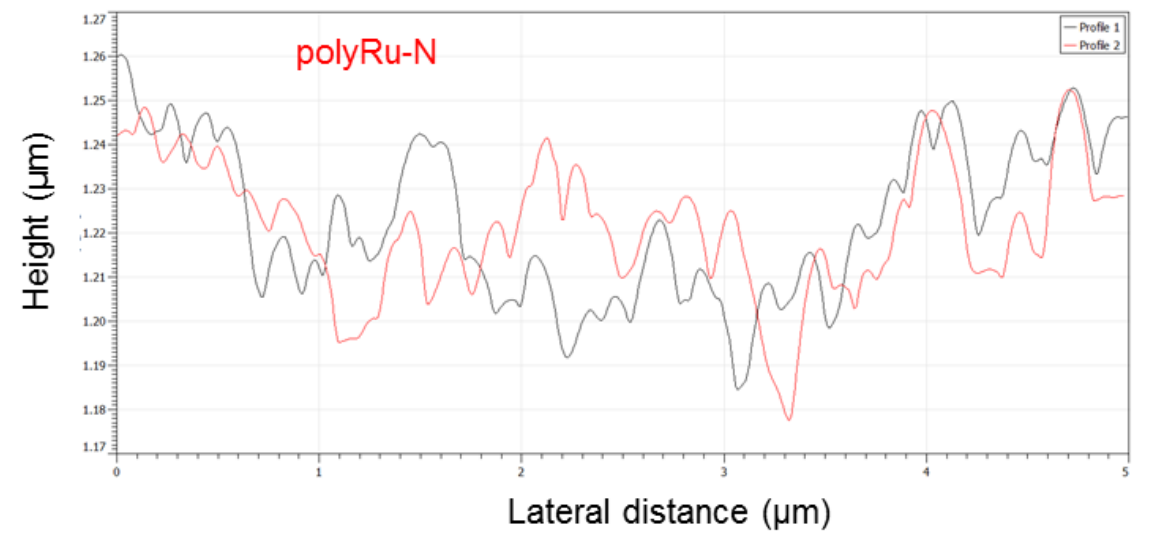

Figure S6. Cross-sectional height profile analysis of AFM topology in (a) polyFe-N and (b) polyRu-N films. 
7. TGA study for polymers

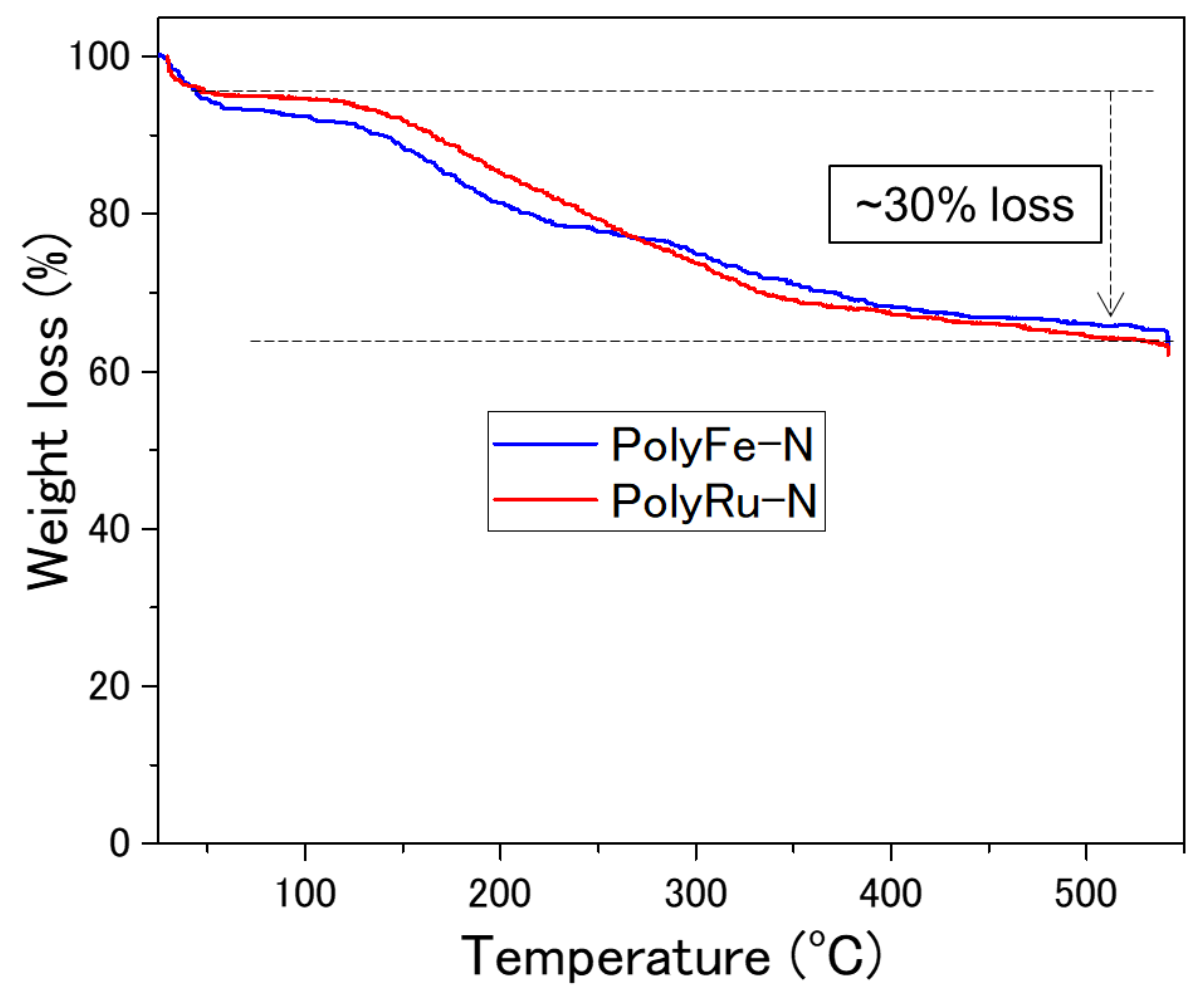

Figure S7. TGA study for polyFe-N and polyRu-N at $\mathrm{N}_{2}$-saturated atmosphere from 25 to $550^{\circ} \mathrm{C}$. 
8. Redox behavior of polymers before/after anneal at $550{ }^{\circ} \mathrm{C}$

(a)

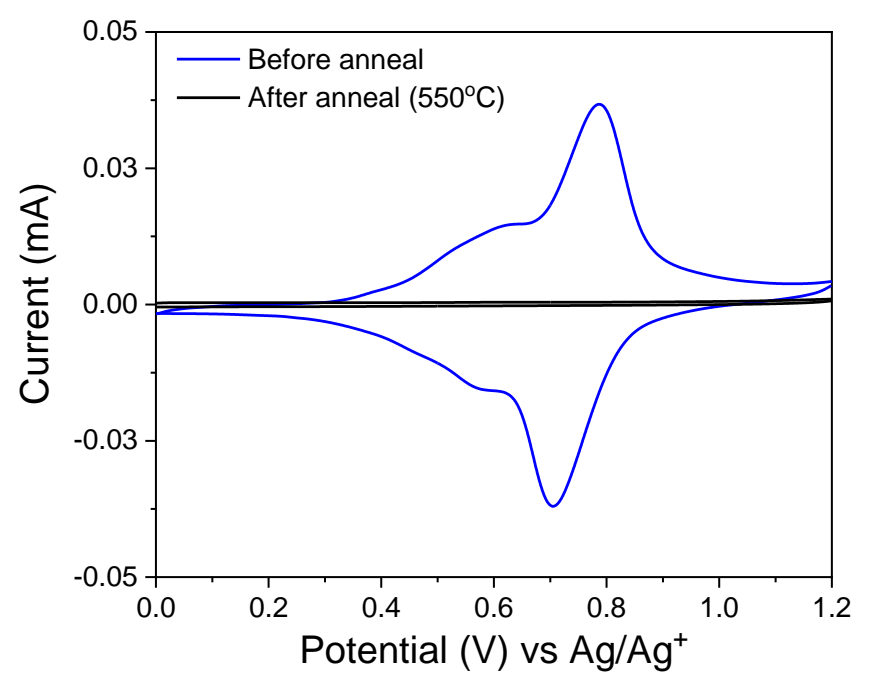

(b)

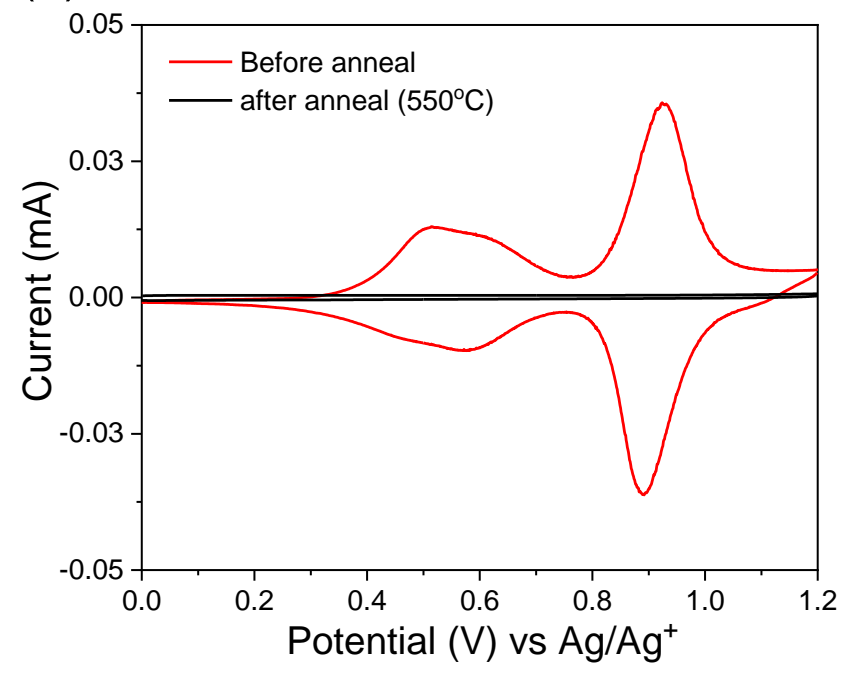

Figure S8. The redox behavior of (a) polyFe-N and (b) polyRu-N before/after annealing at 550 ${ }^{\circ} \mathrm{C}$. 


\section{Different scan rate CV study of polymers}

(a)

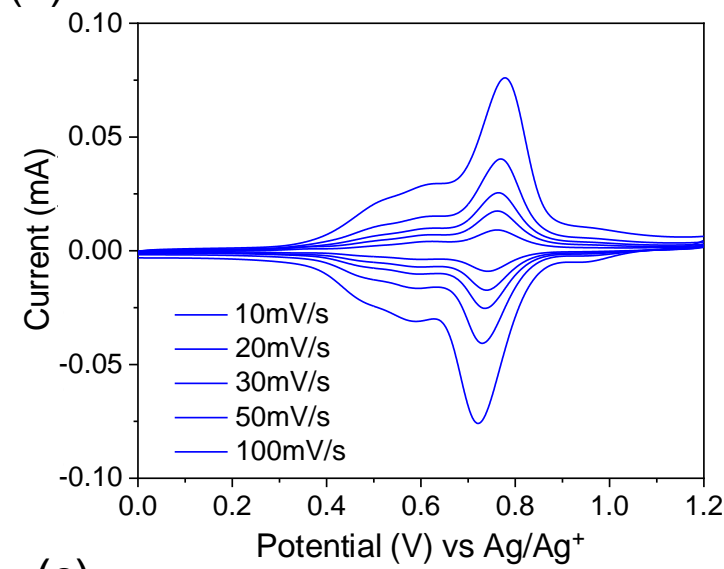

(c)

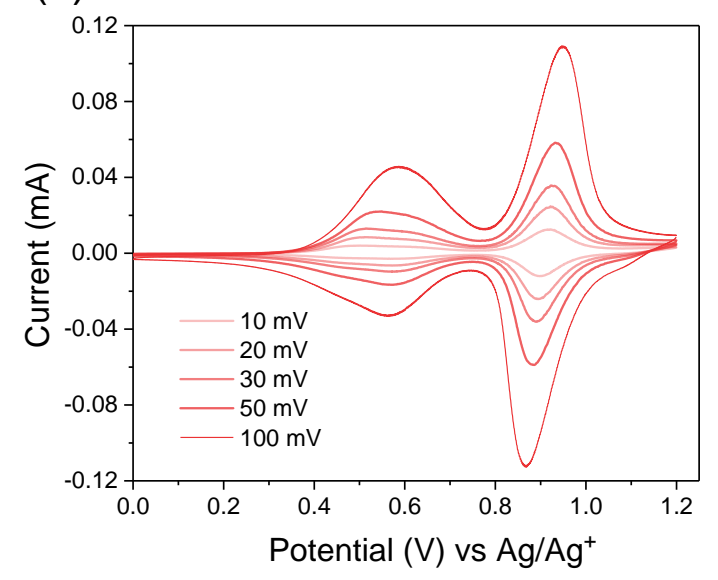

(b)

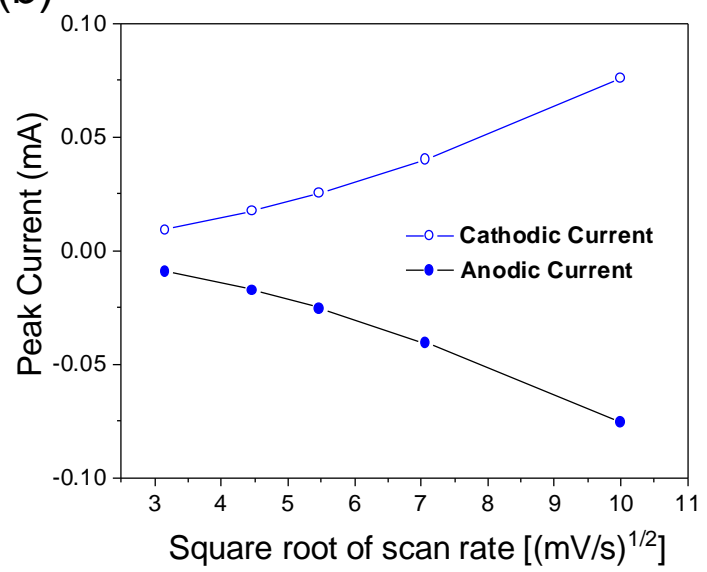

(d)

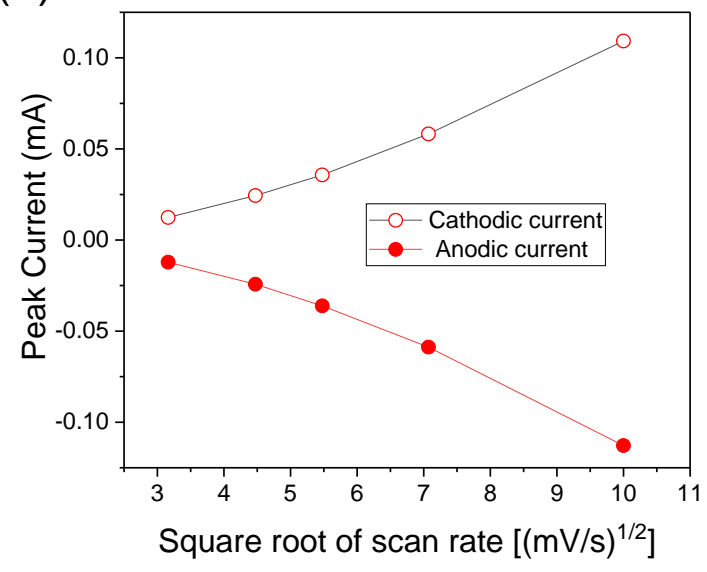

Figure S9. (a) CVs of polyFe-N with different scan speed (10-100 mV/s) and (b) the plots for corresponding peak current versus square root of san rate. (c) CVs of poly Ru-N with different scan speed (10-100 $\mathrm{mV} / \mathrm{s})$ and (d) the plots for the corresponding peak current versus square root of san rate. 
10. Positive and negative peak current charge for both polymers

(a)

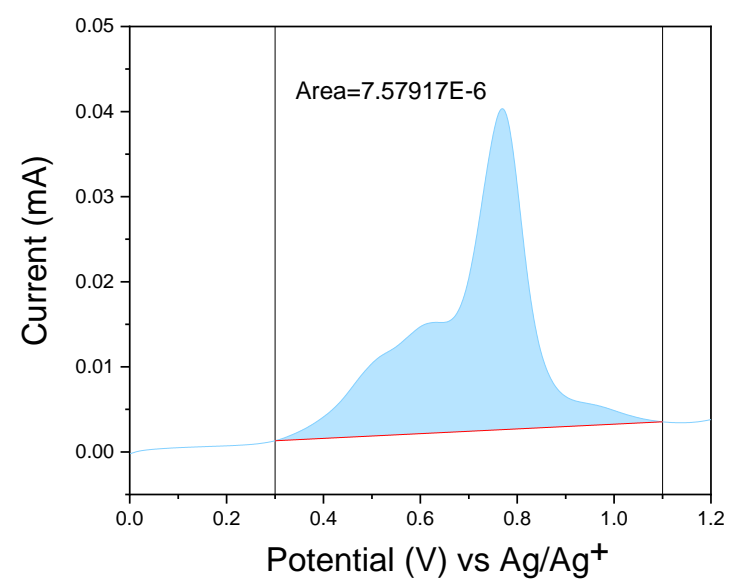

(c)

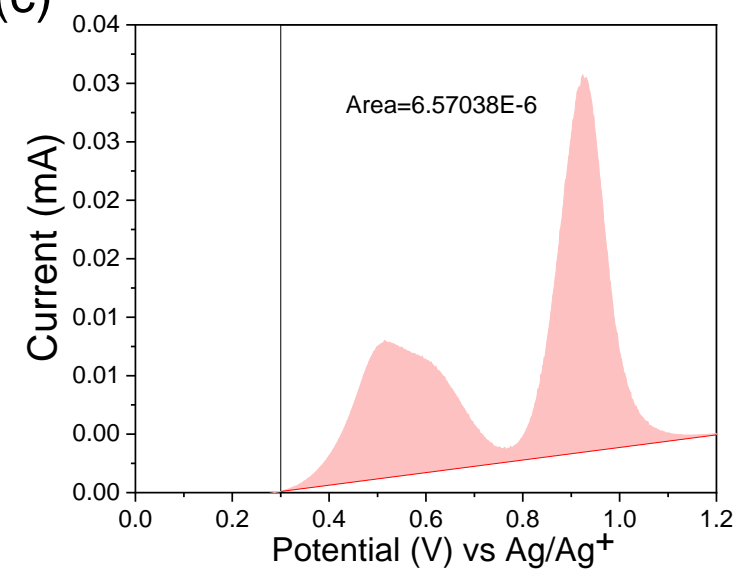

(b)

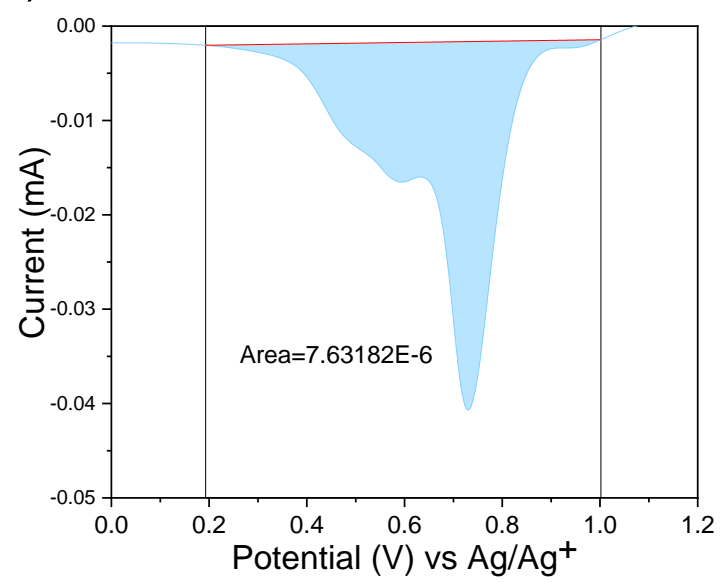

(d)

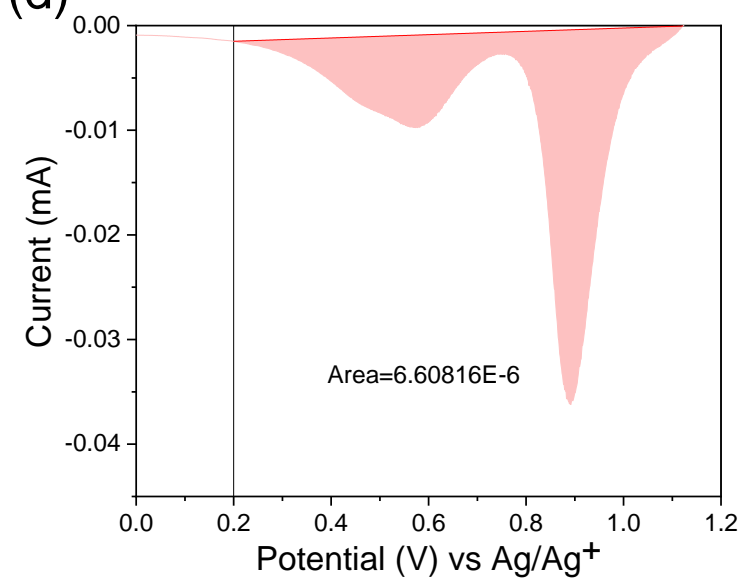

Figure S10. Positive and negative peak current charge of (a, b) polyFe-N and (c, d) polyRu-N. 


\section{Optical contrast in a thick film of polyFe-N}

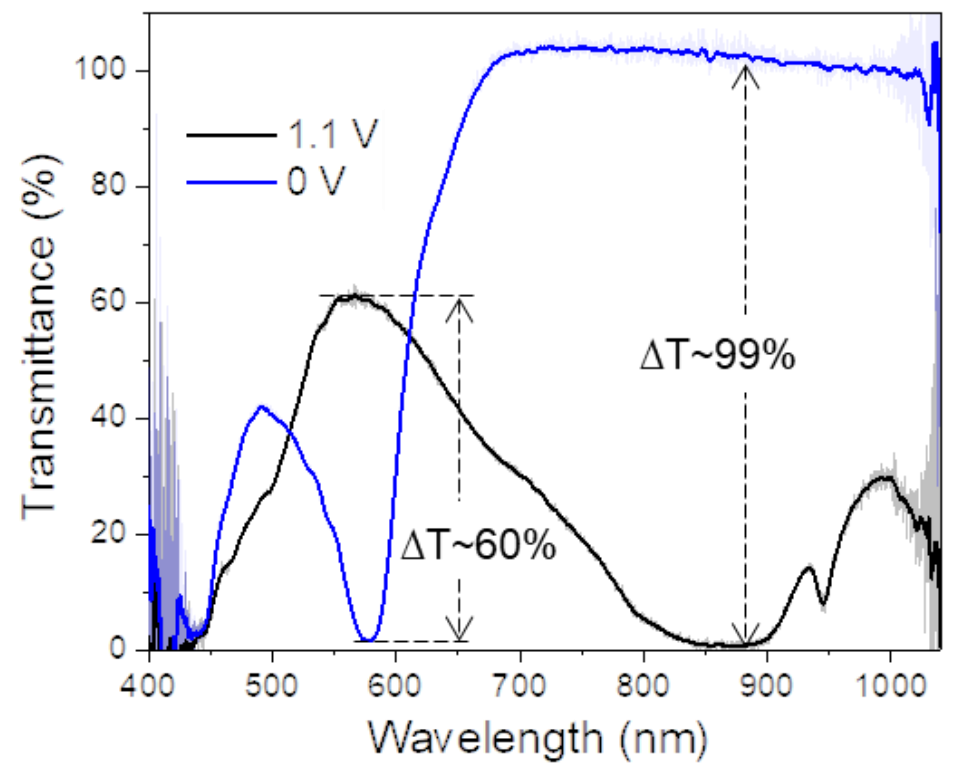

Figure S11. Transmittance changes of a thick film of polyFe-N thick film (applied voltages: 0/1.1V) 
12. Peak current versus time (I-t) of polyFe-N and polyRu-N

(a)

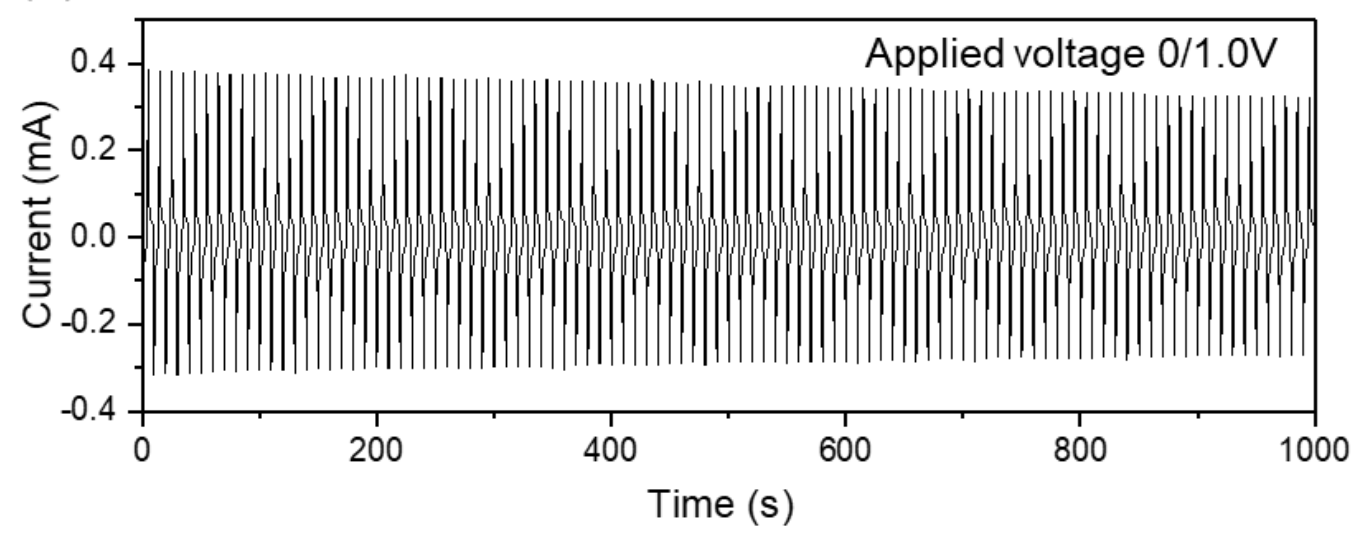

(b)

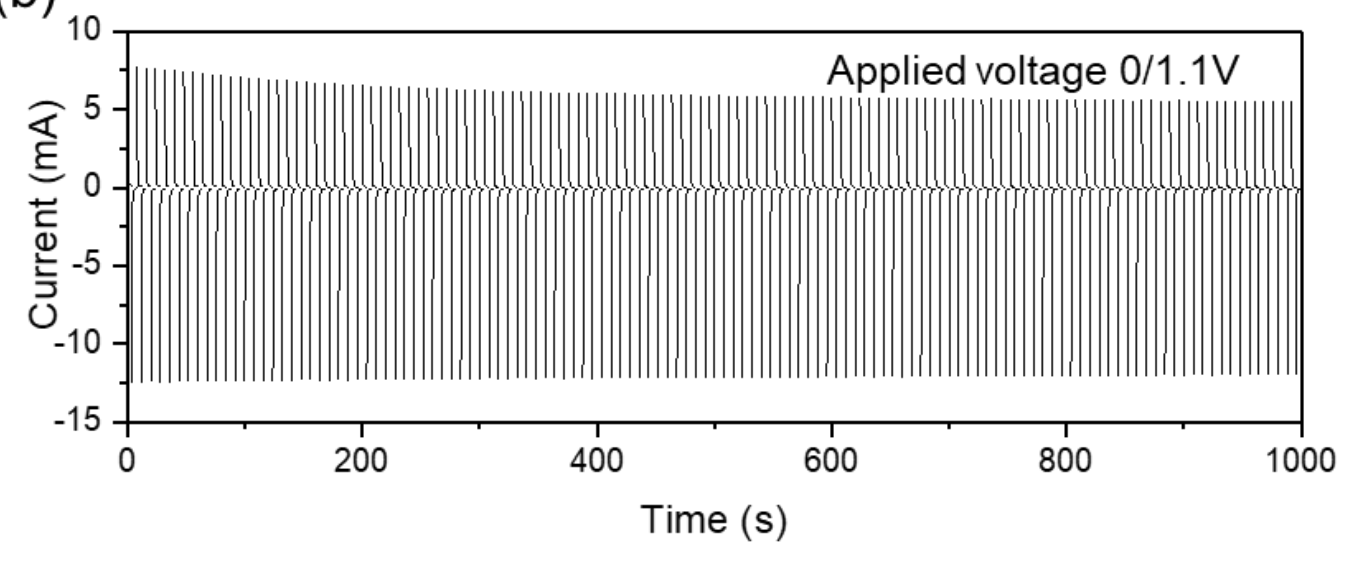

Figure S12. Chronoamperometric behavior for (a) polyFe-N and (b) polyRu-N films within an applied voltage range of $0 / 1.0 \mathrm{~V}$ or $0 / 1.1 \mathrm{~V}$ with $4 \mathrm{sec}$ interval. 


\section{Synthesis of 4,4'-dibromotriphenylamine (4,4'-DBTPA)}

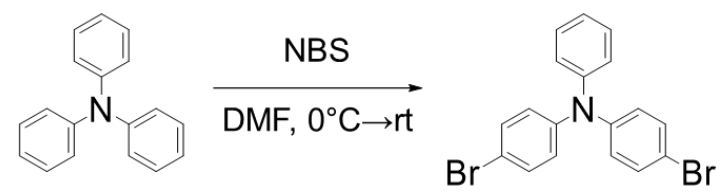

In a flask triphenylamine $(1.23 \mathrm{~g}, 5 \mathrm{mmol})$ and DMF $(12.5 \mathrm{ml})$ were fully mixed and kept in ice bath at $0{ }^{\circ} \mathrm{C}$. A DMF solution $(25 \mathrm{ml})$ of $N$-bromo succinimide (NBS, $1.78 \mathrm{~g}, 10 \mathrm{mmol}$ ) was dropwise added over 30 min to the triphenylamine solution at $0{ }^{\circ} \mathrm{C}$. After an hour ice bath was removed and the resulting reaction mixture was stirred at room temperature for overnight. The same volume of deionized water was added to the resulting reaction mixture with stirring continuously. The product was extracted with $\mathrm{CH}_{2} \mathrm{Cl}_{2}$ and purified by silica gel column chromatography and hexane eluent. The $1.6 \mathrm{~g}$ ( $80 \%$ yield $)$ of the product was obtained. ${ }^{\mathbf{1}} \mathbf{H}-\mathbf{N M R}$ : (400 MHz, $\left.\mathrm{CDCl}_{3}\right), \delta(\mathrm{ppm}): 7.32(\mathrm{dt}, \mathrm{J}=9.5,2.6 \mathrm{~Hz}, 4 \mathrm{H}), 7.25(\mathrm{dd}, \mathrm{J}=8.9,7.1 \mathrm{~Hz}, 2 \mathrm{H}), 7.06-$ 7.03 (m, 3H), 6.94-6.91 (m, 4H) ESI-TOF-MS $\left(\mathrm{CHCl}_{3} / \mathrm{MeOH} 1: 1 \mathrm{v} / \mathrm{v}\right) \mathrm{m} / \mathrm{z}$ : found $402.9(100 \%)$ $400.9(51 \%) 404.9(49 \%)$ for $[\mathrm{M}]^{+}$(calculated M: 400.9 for $\mathrm{C}_{18} \mathrm{H}_{13} \mathrm{Br}_{2} \mathrm{~N}$ ) 


\section{Synthesis of TPyPhBpin}

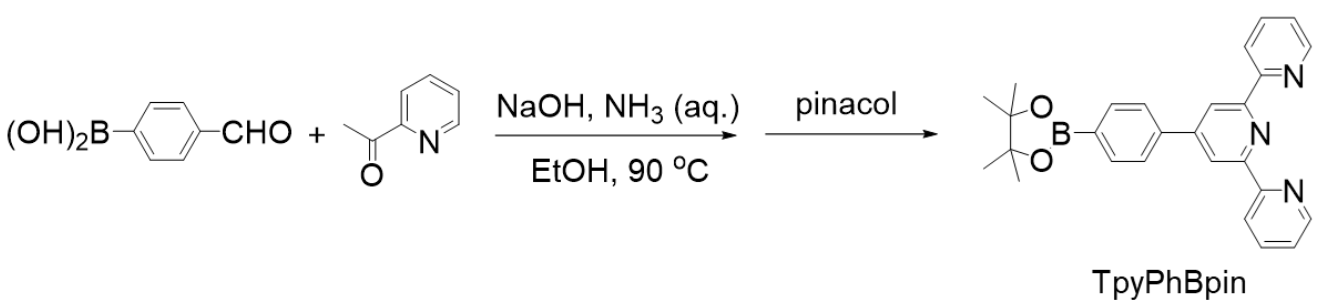

The 4'-(4-(4,4,5,5-tetramethyl-1,3,2-dioxaborolan-2-yl)phenyl)-2,2':6',2"-terpyridine

(TpyPhBpin) was synthesized by Hantzsch pyridine synthesis and pinacol protection procedure. To the suspension of sodium hydroxide (22.5 g, $0.56 \mathrm{~mol}, 7.5$ equiv.) in ethanol (300 mL), 2acetyl-pyridine $(21 \mathrm{ml}, 22.7 \mathrm{~g}, 2.5$ equiv.) was added. The reaction mixture was stirred for $20 \mathrm{~min}$. at $40{ }^{\circ} \mathrm{C}$, then 4-formyl-phenylboronic acid (11.2 g, $75 \mathrm{mmol}, 1.0$ equiv.) and ammonia water $(37 \%, 150 \mathrm{~mL})$ were added. The mixture was continuously stirred for $6 \mathrm{~h}$ at $40^{\circ} \mathrm{C}$ and one day at $90^{\circ} \mathrm{C}$. After cooling to room-temperature, the solid was filtrated and washed with water, dichloromethane to give white solid (19.1 g). To the solid, pinacol (19.2 g, $162 \mathrm{mmol})$, chloroform $(140 \mathrm{~mL})$, and methanol $(10 \mathrm{~mL})$ were added. The mixture was stirred for three days at roomtemperature. The solvent was removed under reduced pressure and the residue was washed by methanol to give the product TPyPhBpin (14.0 g, $32 \mathrm{mmol}, 43 \%)$ as a white solid.

${ }^{1} \mathbf{H}$ NMR (400 MHz, $\mathrm{CDCl}_{3}$ ), $\delta$ (ppm): 8.74-8.73 (m, 4H), 7.95-7.85 (m, 6H), 7.35 (ddd, 2H J = 7.6, 4.8, 1.2 Hz), 1.37 (s, 12H). 